OPEN ACCESS

Edited by:

Zaixu Cui,

University of Pennsylvania

United States

Reviewed by:

Yang Yu,

Second Affiliated Hospital, Zhejiang University School of Medicine, China

Songran Yang,

Sun Yat-sen Memorial Hospital, China

*Correspondence.

Chuancheng Ren

rccfns17@sina.com

${ }^{\dagger}$ These authors have contributed equally to this work

Specialty section: This article was submitted to Brain Imaging Methods, a section of the journal Frontiers in Neuroscience

Received: 28 August 2018 Accepted: 11 December 2018 Published: 07 January 2019

Citation:

Chen J, Sun D, Shi Y, Jin W, Wang Y, Xi Q and Ren C (2019) Dynamic Alterations in Spontaneous

Neural Activity in Multiple Brain

Networks in Subacute Stroke Patients: A Resting-State fMRI Study.

Front. Neurosci. 12:994. doi: 10.3389/fnins.2018.00994

\section{Dynamic Alterations in Spontaneous Neural Activity in Multiple Brain Networks in Subacute Stroke Patients: A Resting-State fMRI Study}

\author{
Jing Chen ${ }^{1 \dagger}$, Dalong Sun ${ }^{2 \dagger}$, Yonghui Shi', Wei Jin ${ }^{1}$, Yanbin Wang ${ }^{3}$, Qian $X^{3}$ and \\ Chuancheng Ren ${ }^{1,4 *}$
}

${ }^{1}$ Department of Neurology, Shanghai Fifth People's Hospital, Fudan University, Shanghai, China, ${ }^{2}$ Division of Gastroenterology, Department of Internal Medicine, Zhongshan Hospital, Fudan University, Shanghai, China, ${ }^{3}$ Department of Radiology, Shanghai East Hospital, Tongji University, Shanghai, China, ${ }^{4}$ Department of Neurology, Shanghai East Hospital, Tongji University, Shanghai, China

Objective: To examine whether subacute stroke patients would exhibit abnormal dynamic characteristics of brain activity relative to healthy controls $(\mathrm{HC})$ and to investigate whether the altered dynamic regional indexes were associated with clinical behavior in stroke patients.

Methods: The dynamic amplitude of low-frequency fluctuations (dALFF) and dynamic regional homogeneity (dReHo) in 42 subacute stroke patients and 55 healthy controls were compared. Correlation analyses between dALFF and dReHo in regions showing significant intergroup differences and clinical scores (i.e., the National Institutes of Health Stroke Scale, Fugl-Meyer assessment and lesion volume size) were conducted in stroke patients. Receiver operating characteristic (ROC) curve analysis was used to determine the potential value of altered dynamic regional indexes to identify stroke patients.

Results: Significantly dALFF in the bilateral cerebellum posterior lobe (CPL), ipsilesional superior parietal lobe, ipsilesional inferior temporal gyrus (ITG), the midline supplementary motor area (SMA), ipsilesional putamen and lentiform nucleus were detected in stroke patients compared to HC. Relative to the HC group, the stroke patients showed significant differences in dReHo in the contralesional rectal gyrus, contralesional ITG, contralesional pons, ipsilesional middle frontal gyrus (MFG). Significant correlations between dALFF variability in midline SMA and Fugl-Meyer assessment (FMA) scores or between dReHo variability in the ipsilesional MFG and FMA scores were detected in stroke patients. Furthermore, the ROC curve revealed that dynamic ALFF at SMA and ReHo at ipsilesional MFG might have the potential to distinguish stroke patients.

Conclusion: The pattern of intrinsic brain activity variability is altered in stroke patients compared with HC, and dynamic ALFF/ReHo might be potential tools to assess stroke patients' motor function.

Keywords: stroke, dynamic intrinsic brain activity, resting-state fMRI, amplitude of low-frequency fluctuations, regional homogeneity 


\section{INTRODUCTION}

Stroke is the most common cause leading to varying degrees of neurological dysfunction with a very high likelihood of long-term disability (Liu et al., 2011; Yang et al., 2013). Movement disorders are the major common conditions of stroke-induced disability, and motor functional recovery remains highly variable. Although the exact mechanism of motor deficits and motor recovery are still under investigation, recent advances in neuroimaging have expanded our understanding. Resting-state functional magnetic resonance imaging (fMRI), which is operationally defined as taskindependent spatiotemporal correlations within functionally related regions of the brain (Biswal et al., 1995), has been extensively used to delineate neural function abnormalities in stroke patients.

Resting-state fMRI measures spontaneous brain activity in low-frequency fluctuations which can be reflected by the blood oxygen level dependent (BOLD) signal. An increasingly large body of resting-state fMRI studies in stroke patients has focused on the characteristics of within-region or interregion functional connectivity, such as connections within motor networks or between motor networks and non-motor networks (Wang et al., 2010; Grefkes and Fink, 2014; Wu et al., 2015). However, few studies have examined regional brain activities in patients with stroke. Neural regional properties are crucial for a better understanding of the neurophysiological and neuropathological conditions, such as regional abnormal energy consumption suggesting excessive or decreased resting metabolic rates (Raichle, 2006; Fox and Raichle, 2007). Currently, one of the methods to measure regional properties of the BOLD signal is the amplitude of low-frequency fluctuations (ALFF), which measures the signal strength in low-frequency oscillations of spontaneous neural activity (Zang et al., 2007). The ALFF is correlated with field potential activity in local brain regions (Logothetis et al., 2001), and the amplitude of oscillations can be applied as an index to examine alterations in neural function (Mohamed et al., 2004). Another approach is regional homogeneity (ReHo), which reflects the statistical similarity of local neural activity among spatially adjacent regions (Zang et al., 2004). These two approaches have been widely adopted for evaluating local neural function in neurologic disorders and neuropsychiatric diseases (Qiu et al., 2011; Li et al., 2012; Liu et al., 2012, 2013).

It has been reported that ALFF or ReHo were altered under resting conditions in stroke patients with movement disorders (Skidmore et al., 2013; Tsai et al., 2014) and that the ALFF value or ReHo value in certain brain regions were associated with the severity of motor deficits (Liu et al., 2015; Zhu et al., 2015). However, the aforementioned investigations of regional brain activities assumed that the BOLD signal is stationary during the entire fMRI scan, ignoring the characteristics of dynamic changes of brain spontaneous activity over time. Indeed, evidence has accumulated that brain responds to internal or external stimuli by dynamic integration or adjustment over multiple time scales (Abrams et al., 2013; Yin et al., 2013). Fortunately, the dynamic nature of brain activity may be detected by task manipulations using methods such as electroencephalography and can also be informed by the lower temporal resolution of resting-state fMRI (Calhoun et al., 2008). In recent years, sliding window approaches to functional connectivity have effectively examined abnormal brain function in stroke (Duncan and Small, 2017). Nevertheless, it is not enough to merely focus on timevarying dynamic functional connectivity, since evidence from neuroimaging techniques of high spatiotemporal resolution has verified that local brain activity itself exists with substantial fluctuations (Liao et al., 2015; Fu et al., 2017), and until now, no study explored the dynamic characteristics of local brain activity indexes in stroke patients. These dynamic local approaches are expected to explore the variability of the oscillation amplitudes and regional synchronization of spontaneous brain activity and to advance our understanding of brain function by identifying specific pathophysiological function signatures and our ability to decipher the neural underpinnings of normal or abnormal human behaviors.

Hence, the present study applied resting-state fMRI to investigate whether subacute stroke patients would exhibit abnormal dynamic characteristics of spontaneous brain activity by calculating regional indexes, ALFF and ReHo, compared with healthy controls (HC). Furthermore, another goal was to explore whether the altered dynamic ALFF (dALFF) and dynamic ReHo (dReHo) were correlated with the clinical behavior of the stroke patients. In the current study, we included subacute stroke patients for two reasons. First, the condition of stroke patients at the subacute stage is relatively stable than acute stroke patients, and the patients' compliance is relatively high, facilitating the smooth progress of the current study. Second, we enrolled stroke patients within 1-3 weeks after symptom onset, and this period is well within the recovery window. A period of dramatic changes in functional and structural reorganization may provide more information of spontaneous neural activity. We hypothesized that variability of regional brain activity was altered in patients with stroke-induced motor deficits compared with $\mathrm{HC}$ and that dynamic regional indexes in certain regions detected to be associated with the Fugl-Meyer assessment (FMA) scores could provide more information for evaluating of motor function in stroke patients.

\section{MATERIALS AND METHODS}

\section{Subjects}

This study was part of an integrated stroke and rehabilitation project at Shanghai 5th People's Hospital affiliated with Fudan University and was approved by the local ethical committee of Shanghai 5th People's Hospital affiliated with Fudan University. Written informed consent was obtained from all subjects before participating according to the Declaration of Helsinki. A total of 45 subacute stroke patients were recruited. Additionally, $55 \mathrm{HC}$, who were right-handed and matched for age, gender and education, were recruited from the local communities. The inclusion criteria for stroke patients were as follows: (1) they were aged 40-80 years; (2) it was a first-onset stroke with a single lesion in right-side subcortical regions as verified by diffusion-weighted imaging (DWI); (3) they were examined within 1-3 weeks after stroke symptom onset; (4) they were clinical evidence of a motor 
deficit based on neurological examination; and (5) they were right-handed before the stroke. The exclusion criteria for both stroke patients and $\mathrm{HC}$ were the presence of any of the following: (1) other brain abnormalities, or clinically significant or unstable medical diseases; (2) unconsciousness, cognitive impairment, or cooperation difficulties; (3) patients with use of medications that could affect motor examination, such as antipsychotics and antiepileptics; (4) patients with cerebellar lesions; and (5) contraindications for MRI scanning. For all stroke patients, the right hemisphere corresponded to the ipsilesional hemisphere. The National Institutes of Health Stroke Scale (NIHSS) and Mini-Mental State Examination (MMSE) were used to evaluate neurological function impairment and cognitive conditions. FMA for upper and lower extremities was applied to evaluate the degree of motor deficit. These clinical behavior scores were collected on the same day as fMRI data acquisition.

\section{Data Acquisition}

All resting-state fMRI data were acquired using a Philips Achieva 3.0 T MR scanner (Philips Medical Systems, Best, Netherlands). Tight but comfortable foam pads and earplugs were used to reduce head motion and scanner noise. Resting-state fMRI was collected using an echo-planar (EPI) sequence with the following scan parameters: repetition time $(\mathrm{TR})=2000 \mathrm{~ms}$; repetition echo time $(\mathrm{TE})=30 \mathrm{~ms}$; flip angle $(\mathrm{FA})=90^{\circ}$; field of view $($ FOV $)=220 \mathrm{~mm} \times 220 \mathrm{~mm}$; voxel size $=3 \mathrm{~mm} \times 3 \mathrm{~mm} \times 3 \mathrm{~mm}$; matrix $=64 \times 64$; slice thickness $=3 \mathrm{~mm}$; gap $=1 \mathrm{~mm}$; interleaved transversal slices $=38$; and number of volumes $=180$. High-resolution sagittal T1-weighted images were acquired using a 3D magnetization prepared rapid gradient echo (MPRAGE) sequence: $\mathrm{TR}=8.0 \mathrm{~ms} ; \mathrm{TE}=3.7 \mathrm{~ms} ; \mathrm{FA}=12^{\circ}$; $\mathrm{FOV}=256 \mathrm{~mm} \times 256 \mathrm{~mm}$; voxel size $=1 \mathrm{~mm} \times 1 \mathrm{~mm} \times 1 \mathrm{~mm}$; matrix $=256 \times 256$; slice thickness $=1 \mathrm{~mm}$; and slices $=180$. During scanning, all participants were instructed to remain awake, keep their eyes closed, and stay motionless without thinking of anything in particular.

The lesion location of each patient was determined by an experienced neuroradiologist on T1-weighted MRI images. We manually outlined the lesion profiles on T1-weighted MRI images slice by slice using the software MRIcron ${ }^{1}$ and generated a lesion mask for each patient. After spatial normalization to Montreal Neurological Institute (MNI) space, all the patients' lesion masks

${ }^{1}$ http://www.mccauslandcenter.sc.edu/mricro/mricron/ overlapped. We then averaged the individual lesion masks and overlaid them with a template to create the lesion overlap map shown in Figure 1.

\section{Preprocessing of Resting-State fMRI Data}

The preprocessing of resting-state fMRI data was performed using the Data Processing Assistant for Resting-State fMRI (DPARSF) version $4.0^{2}$. The first 10 volumes of each participant were deleted to allow the signal to reach equilibrium and the subjects to adapt to the environment. The remaining 170 volumes were corrected for acquisition time delay between slices. Realignment was conducted to correct head motion. The participants with head motion of $>2.0 \mathrm{~mm}$ in maximum displacement or $>2.0^{\circ}$ rotation in angular motion were excluded from the study. The mean framewise displacement (FD) was computed by averaging the FD of each subject across the time points, and no significant differences were found between stroke patients and $\mathrm{HC}(p=0.235)$. In addition, each subject's mean FD was included in all group-level analyses as a covariate to further control the head move effect. Subsequently, the structural image was coregistered to the mean functional image after the motion correction, and the transformed structural image was segmented into gray matter, white matter, and cerebrospinal fluid. Then, the segmented images were normalized to MNI space using Diffeomorphic Anatomical Registration Through Exponentiated Lie algebra (DARTEL) algorithm (Ashburner, 2007). Next, the motion-corrected functional volumes were normalized to the MNI space using the normalization parameters for their respective structure images and resampled into a voxel size of $3 \mathrm{~mm} \times 3 \mathrm{~mm} \times 3 \mathrm{~mm}$. Nuisance covariates (24 head motion parameters, cerebrospinal fluid signal, white matter signal and linear trend) were regressed out. Given that it is still a controversy of removing the global signal (Murphy et al., 2009); we did not regress out the global signal. For the ReHo calculation, an additional processing step was that the regressed functional images were temporally bandpass filtered $(0.01-0.08 \mathrm{~Hz})$ to reduce low frequency drift and high-frequency noise.

\section{dALFF and dReHo Analysis}

Dynamic regional metrics analysis was performed using Temporal Dynamic Analysis (TDA) toolkits based on DPABI

${ }^{2}$ http://rfmri.org/DPARSF/

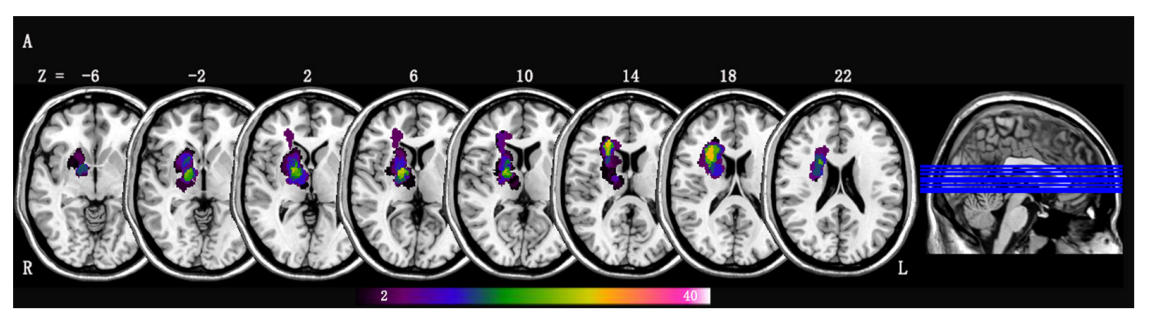

FIGURE 1 | Lesion overlap map across stroke patients with right-sided lesions ( $n=42)$; Lesion maps were normalized to an MNI reference brain. The color bar indicates the percentage of lesion overlap. 
(Yan et al., 2017). Sliding window-based analysis, which is sensitive in detecting time-dependent variations (Hindriks et al., 2016; Liu F. et al., 2017; Yip et al., 2017), was applied to examine the dALFF or dReHo variability over the whole brain. In the sliding window analysis, a temporal window of certain size and shape is chosen, and ALFF and ReHo within that window are calculated. Ideally, the window size should be small enough to detect potentially transient signals, and yet large enough to analyze the lowest frequencies of interest in the signals (Sakoglu et al., 2010). Previous work of sliding window connectivity have applied a sliding window length of as small as $10 \mathrm{~s}$ (Thompson et al., 2013) and as long $180 \mathrm{~s}$ (Gonzalez-Castillo et al., 2015). In this work, a moderate-length sliding window of 32 TR (64 s) and a shifting step size of one TR (2 s) were used to simultaneously maximize statistical power within the window and also maximize statistical power for cross-level analyses (Allen et al., 2014). The remaining 170 time points after removing the first 10 time points for each individual were segmented into 139 windows in total. In each sliding window, ALFF and ReHo were calculated. For ALFF, the time series was first converted to the frequency domain using a fast Fourier transform, and then the ALFF value of a given voxel was obtained by calculating and summing the square root of the power spectrum between 0.01 and $0.08 \mathrm{~Hz}$. For ReHo, the Kendall's coefficient of concordance (KCC) of the time course of every 27 nearest neighboring voxels was calculated (Zang et al., 2004). The standard deviation (SD) of ALFF values and ReHo values at each voxel across 139 windows was calculated to assess the variability of ALFF and ReHo. To reduce the global effects of variability across subjects, the dALFF and $\mathrm{dReH}$ o of each voxel were divided by the global mean dALFF and dReHo values within a gray matter mask, respectively. Finally, the mean normalized dALFF and dReHo maps were spatially smoothed with an isotropic Gaussian kernel of $4 \mathrm{~mm}$ full-width-at-half-maximum (FWHM).

\section{Statistical Analysis}

A general linear model (GLM) was used in a voxel-wise manner to compare group differences between the stroke group and HC group in dALFF and dReHo with age, gender, educational level, MMSE and mean FD as covariates. Multiple comparisons were corrected using a voxel-level familywise error rate (FWE) method with corrected $p<0.05$ (cluster size $\geq 50$ voxels).

The Shapiro-Wilk statistic was first used to test for normality, and then group comparisons of clinical measures were performed using two-sample $t$-tests for continuous data and Pearson's chi-squared test for categorical data. Partial correlation analyses were conducted in stroke patients between the clinical measures (NIHSS scores, FMA scores and lesion volume size) and the mean dALFF/dReHo value of each cluster showing significant group differences between stroke group and $\mathrm{HC}$ group. The age, gender, educational level, MMSE, mean FD, illness duration and intravenous thrombolysis (IVT) were also considered as covariates. As the correlation analyses were exploratory in nature, the significance levels were set at uncorrected $p<0.05$. Furthermore, receiver operating characteristic (ROC) curve analysis was performed to examine the potential value of altered dynamic ALFF or ReHo values in clusters showing significant correlations with clinical behaviors in stroke patients. The optimal cut-off between sensitivity and specificity was determined by maximizing the Youden's index J $(J=$ sensitivity + specificity -1$)$. A two-tailed $p$-value of 0.05 was considered statistically significant for the analyses conducted with SPSS version 19.0 statistical software (IBM Corporation, Armonk, NY, United States).

\section{RESULTS}

\section{Clinical Data}

Data obtained from three stroke patients were excluded because of excessive head motion during scanning. Demographic and clinical characteristics of 42 patients with right hemisphere stroke (21 men; mean age, $57.86 \pm 11.17$ years) and $55 \mathrm{HC}$ (29 men; mean age, $56.73 \pm 10.21$ years) are listed in Table 1. No significant differences in were found $(p>0.05)$ in gender, age, education level, high risk factors (hypertension, diabetes, hyperlipidemia and atrial fibrillation) and MMSE scores between the stroke group and HC group. The mean lesion volume of stroke patients was $6.10 \pm 8.55 \mathrm{cc}^{3}$. Among them, four patients received IVT therapy. Of the 42 patients, 15 had corona radiate lesions, 15 had internal capsule lesions, 10 had basal ganglia lesions, and two had thalamus lesions. The lesion overlaps of stroke patients are shown in Figure 1.

TABLE 1 | Demographic and clinical data.

\begin{tabular}{|c|c|c|c|c|}
\hline & $\begin{array}{l}\text { Stroke patients } \\
\qquad(n=42)\end{array}$ & $\begin{array}{l}\text { Healthy control } \\
\qquad(n=55)\end{array}$ & $t / \chi^{2}$ & $p$ \\
\hline Gender, male & 21 (50.0) & $29(52.7)$ & 0.071 & 0.790 \\
\hline Age, years & $57.86 \pm 11.17$ & $56.73 \pm 10.21$ & 0.518 & 0.605 \\
\hline $\begin{array}{l}\text { Educational level, } \\
\text { years }\end{array}$ & $10.74 \pm 3.39$ & $11.00 \pm 3.82$ & -0.351 & 0.727 \\
\hline \multicolumn{5}{|l|}{ High risk factor } \\
\hline Hypertension & $25(59.5)$ & $29(52.7)$ & 0.446 & 0.504 \\
\hline Diabetes & $11(26.2)$ & $12(21.8)$ & 0.252 & 0.616 \\
\hline Hyperlipidemia & $12(28.6)$ & $16(29.1)$ & 0.003 & 0.955 \\
\hline Atrial fibrillation & $2(2.1)$ & $1(1.8)$ & 0.689 & 0.407 \\
\hline MMSE & $28.07 \pm 1.28$ & $28.15 \pm 1.27$ & -0.284 & 0.777 \\
\hline Illness duration, days & $14.29 \pm 2.14$ & - & & \\
\hline \multicolumn{5}{|l|}{ Stroke type } \\
\hline Ischemia & 40 (95.2) & - & & \\
\hline Hemorrhage & $2(4.8)$ & - & & \\
\hline \multicolumn{5}{|l|}{ Location of lesion } \\
\hline Coronal radiate & $15(35.7)$ & - & & \\
\hline Internal capsule & $15(35.7)$ & - & & \\
\hline Basel ganglia & $10(23.8)$ & - & & \\
\hline Thalamus & $2(4.8)$ & - & & \\
\hline Lesion volume, $\mathrm{cc}^{3}$ & $6.10 \pm 8.55$ & - & & \\
\hline NIHSS & $5.86 \pm 4.14$ & - & & \\
\hline FMA-total & $76.45 \pm 14.12$ & - & & \\
\hline IVT use & $4(9.5)$ & - & & \\
\hline
\end{tabular}

Data represent $n$ (\%) or mean $\pm S D$. MMSE, the Mini-Mental State Examination; NIHSS, the National Institutes of Health Stroke Scale; FMA-total, Fugl-Meyer assessment for upper and lower extremities; IVT, intravenous thrombolysis. 


\section{Differences in Dynamic ALFF and Dynamic ReHo}

The significant differences in dALFF and dReHo between the stroke group and HC group are shown in Figures 2A,B, respectively. Compared with HC, significantly increased dALFF variability in the contralesional cerebellum posterior lobe (CPL), ipsilesional superior parietal lobe, ipsilesional inferior temporal gyrus (ITG), ipsilesional CPL and cerebellum tonsil and decreased dALFF variability in the midline supplementary motor area (SMA), ipsilesional putamen and lentiform nucleus were detected in stroke patients (Table 2 and Figures 2A,C). Relative to the dReHo variability of subjects in the HC group, these stroke patients showed a significant increase in contralesional rectal gyrus, contralesional ITG, contralesional pons and a significant decrease in ipsilesional middle frontal gyrus (MFG) (Table 3 and Figures 2B,D).

\section{Correlational Analysis}

A significant positive correlation was detected between the FMA scores and dALFF variability in the SMA $(r=0.347$, $p=0.035$, uncorrected; Figure $3 \mathrm{~A}$ ), a significant negative correlation between the FMA scores and dReHo variability in the midline ipsilesional MFG was found in stroke patients $(r=-0.462, p=0.004$, uncorrected; Figure 3B). No other significant correlations between NIHSS, size of lesion volume and dynamic regional indexes were observed in the stroke group.

\section{ROC Analysis}

As shown above, significant correlations were detected between the FMA scores and dynamic ALFF variability in SMA or dynamic ReHo variability in ipsilesional MFG, which proposed that the dynamic ALFF/ReHo in these regions might be utilized to differentiate the stroke patients from healthy persons. To verify this possibility, mean $\mathrm{dALFF} / \mathrm{dReHo}$ values in the SMA or ipsilesional MFG were extracted. Then, ROC analysis was performed to investigate this possibility. The results demonstrated that the area under the curves (AUC) of SMA and ipsilesional MFG were 0.965 and 0.911, respectively (Figure 4), which suggested that dynamic ALFF in SMA and ReHo values in the ipsilesional MFG might have the potential to distinguish the stroke patients from healthy subjects. Further diagnostic analysis showed that the sensitivity and specificity were relatively high (Table 4).
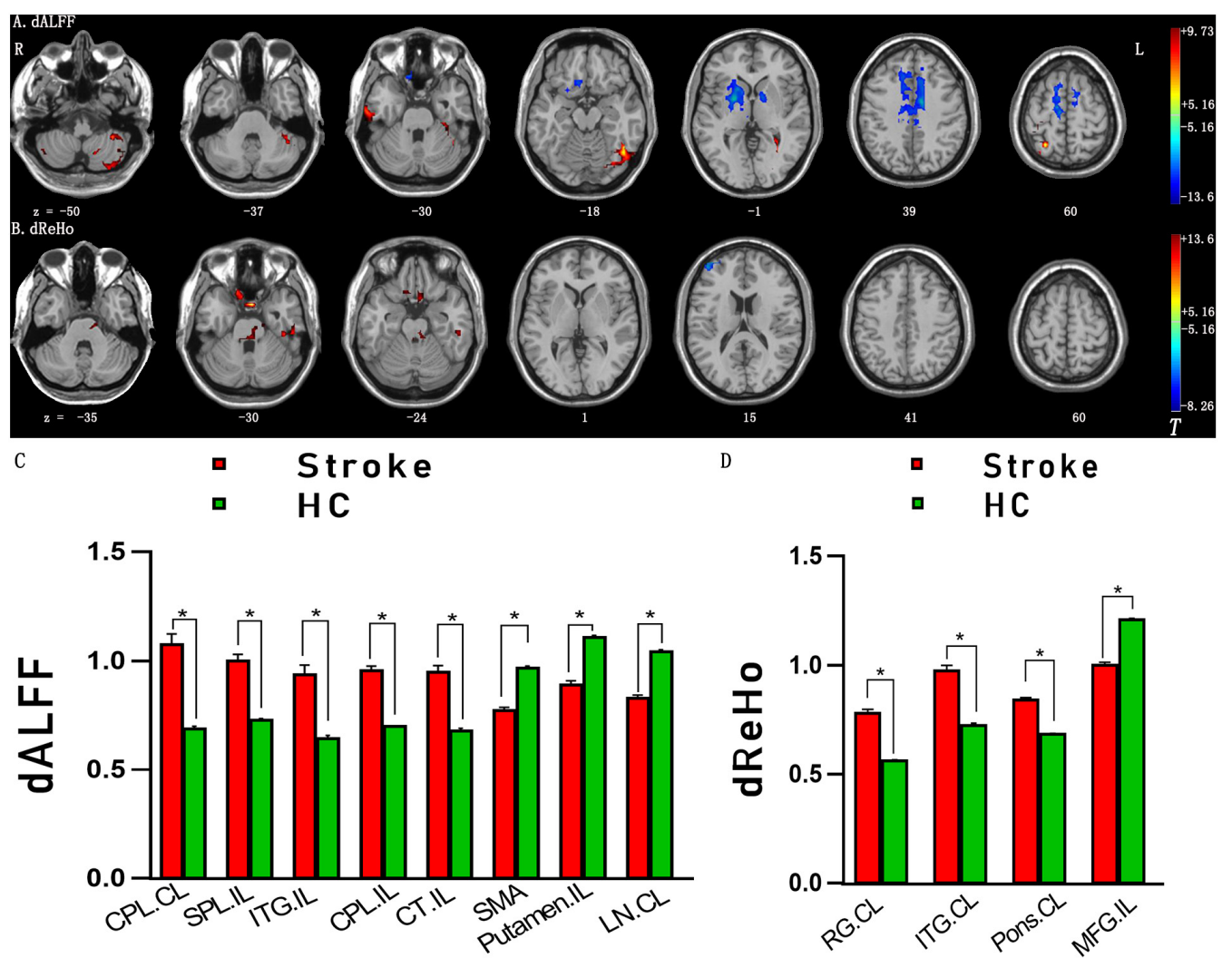

FIGURE 2 | (A) Brain regions with significant intergroup differences in dALFF between the stroke group and HC group. (B) Brain regions with significant intergroup differences in $\mathrm{dReHo}$ between the stroke group and $\mathrm{HC}$ group. (C) The histogram indicates mean values and standard error of dALFF variability between the stroke group and HC group. (D) The histogram indicates mean value and standard error of dReHo variability between the stroke group and HC group. Familywise error rate corrected $\left(p<0.05\right.$, cluster size $\geq 50$ voxels). The color bar indicates the $T$ value. ${ }^{*} p<0.05$. HC, healthy controls; dALFF, dynamic amplitude of low-frequency fluctuation; dReHo, dynamical regional homogeneity; CPL, cerebellum posterior lobe; SPL, superior parietal lobe; ITG, inferior temporal gyrus; CT, cerebellum tonsil; SMA, supplementary motor area; LN, lentiform nucleus; RG, rectal gyrus; MFG, middle frontal gyrus; IL, ipsilesional; CL, contralesional. 
TABLE 2 | Brain regions with significant differences in dynamic ALFF between groups.

\begin{tabular}{|c|c|c|c|c|c|c|}
\hline \multirow[b]{2}{*}{ Group comparisons } & \multirow[b]{2}{*}{ Brain regions/BA } & \multicolumn{3}{|c|}{ Peak MNI coordinates } & \multirow[b]{2}{*}{ Cluster size (voxels) } & \multirow[b]{2}{*}{ Peak $t$ values } \\
\hline & & $\mathbf{x}$ & $\mathbf{Y}$ & $\mathbf{Z}$ & & \\
\hline \multirow[t]{5}{*}{ Stroke patients $>\mathrm{HC}$} & Cerebellum posterior lobe, CL & -15 & -69 & -39 & 1175 & 9.73 \\
\hline & Superior parietal lobe, IL/4 & 27 & -57 & 60 & 179 & 9.32 \\
\hline & Inferior temporal gyrus, IL/20 & 39 & -57 & -18 & 298 & 9.27 \\
\hline & Cerebellum posterior lobe, IL & 39 & -87 & -39 & 53 & 8.33 \\
\hline & Cerebellum tonsil, IL & 48 & -63 & -54 & 50 & 6.57 \\
\hline \multirow[t]{3}{*}{ Stroke patients < HC } & Supplementary motor area, 6 & 12 & -12 & 57 & 1670 & 13.6 \\
\hline & Putamen, IL & 24 & 0 & 0 & 814 & 12.18 \\
\hline & Lentiform nucleus, CL & -18 & -6 & 18 & 298 & 11.01 \\
\hline
\end{tabular}

ALFF, amplitude of low-frequency fluctuation; HC, healthy controls; BA, Brodmann's area; MNI, Montreal Neurological Institute; IL, ipsilesional; CL, contralesional.

TABLE 3 | Brain regions with significant differences in dynamic ReHo between groups.

\begin{tabular}{|c|c|c|c|c|c|c|}
\hline \multirow[b]{2}{*}{ Group comparisons } & \multirow[b]{2}{*}{ Brain regions/BA } & \multicolumn{3}{|c|}{ Peak MNI coordinates } & \multirow[b]{2}{*}{ Cluster size (voxels) } & \multirow[b]{2}{*}{ Peak $t$ values } \\
\hline & & $\mathbf{X}$ & $\mathbf{Y}$ & $\mathbf{Z}$ & & \\
\hline \multirow[t]{3}{*}{ Stroke patients > HC } & Rectal gyrus, CL/11 & -3 & 6 & -30 & 105 & 13.16 \\
\hline & Inferior temporal gyrus, CL/20 & -42 & -30 & -30 & 58 & 8.85 \\
\hline & Pons, CL & -12 & -21 & -33 & 95 & 7.86 \\
\hline Stroke patients < HC & Middle frontal gyrus, IL/9 & 45 & 54 & 18 & 52 & 7.32 \\
\hline
\end{tabular}

ReHo, regional homogeneity; HC, healthy controls; BA, Brodmann's area; MNI, Montreal Neurological Institute; IL, ipsilesional; CL, contralesional.

A

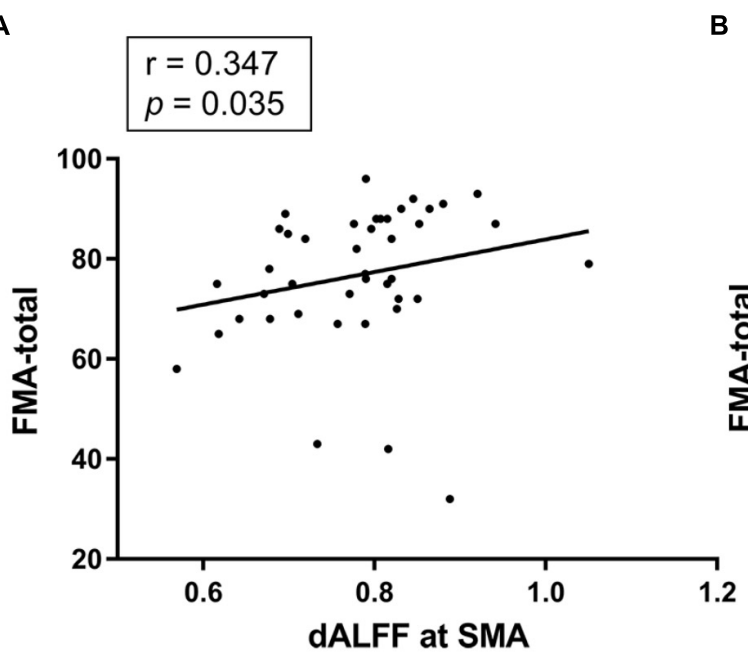

B

$$
\begin{aligned}
& r=-0.462 \\
& p=0.004
\end{aligned}
$$

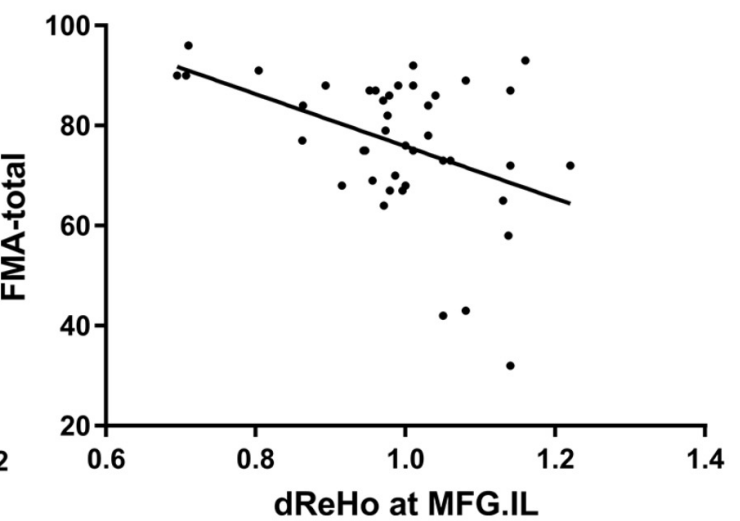

FIGURE 3 | (A) The dALFF variability in the SMA was positively correlated with the FMA scores of the stroke patients $(r=0.347, p=0.035$, uncorrected). (B) The dReHo variability in the ipsilesional MFG was negatively correlated with the FMA scores of the stroke patients $(r=-0.462, p=0.004$, uncorrected). dALFF, dynamic amplitude of low-frequency fluctuation; dReHo, dynamic regional homogeneity; FMA-total, Fugl-Meyer assessment for upper and lower extremities; SMA,

supplementary motor area; MFG, middle frontal gyrus; IL, ipsilesional.

\section{DISCUSSION}

In the present study, dynamic regional brain activity between stroke patients with motor deficits and healthy controls was examined using resting-state fMRI. To the best of our knowledge, there is no prior study using a TDA approach to detect strokerelated brain activity changes in humans. Given that young adult stroke could be different both in causes and in outcomes
(Edwards et al., 2018) and the role of educational level in recovery should not be ignored (Hillis and Tippett, 2014). Therefore, we used age and educational level as covariates in intergroup difference analyses and correlation analyses. Moreover, patients were enrolled in this current study within 1-3 weeks after stroke symptom onset, during which period imaging data and behavior performance were obtained. This period is well within the recovery window for stroke (Venketasubramanian et al., 2017). 


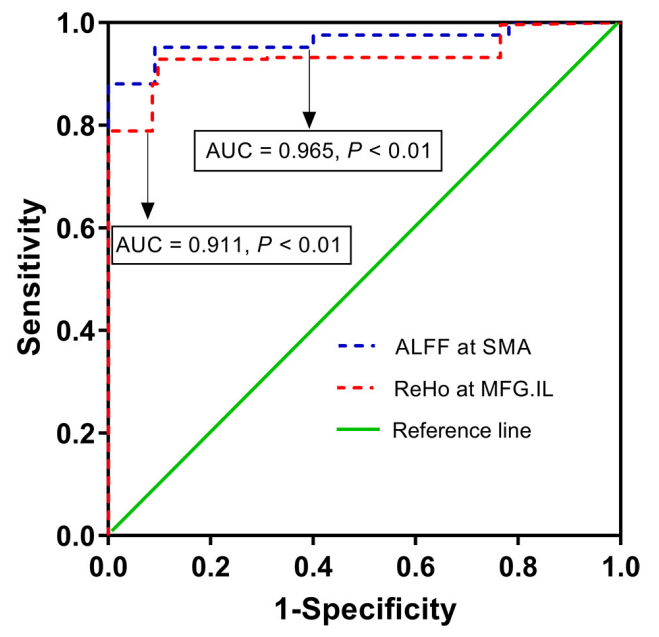

FIGURE 4 | The diagnostic performance of altered dynamic ALFF in SMA and dynamic ReHo in ipsilesional MFG in distinguishing stroke patients from healthy subjects. ALFF, amplitude of low-frequency fluctuation; ReHo, regional homogeneity; SMA, supplementary motor area; MFG, middle frontal gyrus; IL, ipsilesional; AUC, area under curve.

Additionally, medication, especially IVT therapy, can largely improve patients' functional outcomes for hyperacute ischemic stroke (Ferrigno et al., 2018). Hence, we also considered patients' illness duration and IVT use status as covariates in correlation analyses to reduce the confounding effects.

Differences in dALFF variability were observed between the stroke group and HC group in the contralesional CPL, ipsilesional superior parietal lobe, ipsilesional ITG, ipsilesional CPL, cerebellum tonsil, the midline SMA, ipsilesional putamen and lentiform nucleus, while differences of dReHo variability in contralesional rectal gyrus, contralesional ITG, contralesional pons and MFG distinguished stroke patients from HC. In addition, relationships were observed between the FMA scores and dynamic ALFF or ReHo variability in SMA or ipsilesional MFG in stroke patients. Further ROC analyses suggested that dynamic ALFF in SMA or ReHo in ipsilesional MFG had the potential to distinguish the patients of subacute stroke from healthy subjects.

Our findings were in line with previous studies that investigated stroke patients with movement disorders by functional connectivity, structural connectivity or regional metrics, such as ALFF or ReHo (Skidmore et al., 2013; Tsai et al., 2014; Zhu et al., 2015). The sensorimotor network (SMN), which is critical for voluntary movement, connects primary motor cortex function with SMA function (Damoiseaux et al., 2006; Chang et al., 2013). The SMA is a part of the primate cerebral cortex that contributes to the control of movement. Neurons in the SMA project directly to the spinal cord and may play a role in the direct control of movement (Nudo and Masterton, 1990). It has been reported that SMA plays roles in the postural stabilization of the body, the coordination of both sides of the body such as during bimanual action, the control of movements that are internally generated rather than triggered by sensory events, and the control of sequences of movements (Shima and Tanji, 1998; Cunnington et al., 2003; Zhang et al., 2012). Stroke-induced disturbance of intrinsic neural activity, which may be due to a complex cascade of events that are associated with structural reorganization and axonal sprouting as demonstrated by tract-tracing studies in animal models of stroke (Carmichael et al., 2001; Dancause et al., 2005), impedes brain network function of voluntary motion. Based on prior studies, we speculate that altered dALFF and dReHo variability in the SMN (e.g., in the superior parietal lobe and SMA) of stroke patients with motor deficits might occur as a compensatory mechanism and might be a significant factor in the reorganization and integration of resting-state functional networks at the subacute stage.

We observed decreased dReHo variability in the ipsilesional MFG, which belongs to the default mode network (DMN). This finding may indicate that stroke-related brain activity changes not only occurred in motor-related areas but also in non-motor regions. The DMN plays a pivotal role in "resting" brain activity, which is involved in sustaining attention, self-consciousness and exhibiting self-control (Andrews et al., 2011; Yuan et al., 2018). In stroke patients, alterations in brain activity of the DMN may be associated with advanced neural function of cognitive and emotional control (Liu F. et al., 2017). Evidence has shown that the DMN regulates consciousness, processes emotionally salient stimuli, and coordinates the interactions of cognitive function and emotional processing (Soddu et al., 2012; Zhang et al., 2017). In the current study, we demonstrated that the stroke group showed significantly decreased ReHo variability in the ipsilesional MFG, indicating that the synchronous neural activity was also disrupted in the ipsilesional MFG in stroke patients.

The increased regional brain activity in contralesional pons and bilateral cerebellum CPL, ipsilesional cerebellum tonsil may

TABLE 4 | ROC analysis for differentiating stroke patients from healthy person.

\begin{tabular}{|c|c|c|c|c|c|c|c|}
\hline \multirow[b]{2}{*}{ Brain regions } & \multicolumn{3}{|c|}{ MNI coordinates } & \multirow[b]{2}{*}{ AUC } & \multirow[b]{2}{*}{ Maximal Youden's index $\mathrm{J}$} & \multirow[b]{2}{*}{ Sensitivity } & \multirow[b]{2}{*}{ Specificity } \\
\hline & $x$ & $\mathbf{Y}$ & $\mathbf{Z}$ & & & & \\
\hline dALFF at SMA & 12 & -12 & 57 & 0.965 & $0.861^{*}$ & $95.2 \%(40 / 42)$ & $90.9 \%(50 / 55)$ \\
\hline dReHo at MFG.IL & 45 & 54 & 18 & 0.911 & 0.832 & $92.9 \%(39 / 42)$ & $90.3 \%(49 / 55)$ \\
\hline
\end{tabular}

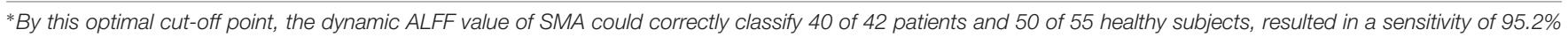

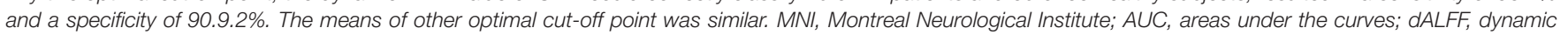
amplitude of low-frequency fluctuation; dReHo, dynamic regional homogeneity; SMA, supplementary motor area; MFG, middle frontal gyrus; IL, ipsilesional. 
result from the dysfunction of the cerebro-ponto-cerebellar circuit and act as a compensatory response (Lu et al., 2011). The excessive ReHo variability of the prefrontal cortex (the rectal gyrus) may compensate for the deficits in motor function in stroke patients. However, this concept needs to be confirmed. Moreover, rectal gyrus is considered as a part of affective network (AN). Study has reported that abnormal regional neural activity was observed mainly in component of DMN, SMN, cerebellar lobes (CPL) and AN in stroke patients with emotional abnormality (Liu T. et al., 2017). Hence, we tend to speculate that the dysfunction of the volitional system may lead to the disorder of emotional system in stroke patients.

We also found decreased dALFF variability in the ipsilesional putamen and contralesional lentiform nucleus in stroke patients. Considered the entrance to the basal ganglia, the lentiform nucleus receives the input from cerebral cortex, and there was partial stroke lesion overlap observed in the present study and shown in Figure 1. The primary roles of these regions are to regulate movements at various stages, such as during motor preparation and motor execution. Meanwhile, they play important roles in motor learning, which can be considered a sensory feedback of frontal mediated goal parameters and posterior-mediated motor programs; there are anatomical links that exist between these motor-execution and frontal-parietal motor control systems (Ween, 2008). Previous human and nonhuman studies of stroke models have shown that decreased ALFF values were found in the core of stroke lesions (Liu et al., 2007; Skidmore et al., 2013). The mechanism may involve abrupt decreases of the blood flow resulting from cytotoxic swelling, calcium overload and membrane ion pump failure in core regions of stroke lesions (Badaut et al., 2011). Based on the previous studies and this present study, we infer that deceased or vanished oxygen consumption and intrinsic brain activity may contribute to less variation and low ALFF values.

In addition, we also detected excessive intrinsic brain activity variability reflected both in dALFF in the ipsilesional ITG and in dReHo in the contralesional ITG of the stroke patients. The ITG belongs to the higher levels of the ventral stream of visual processing and is related to the representation of complex object features. A previous study found that the visual network was activated in recovery from sensorimotor stroke, and limb movement critically relies on visual guidance (Archer et al., 2016). The mechanism underlying the abnormal brain activity of contralesional ITG is presently poorly understood.

The current study has several limitations. First, although we identified significant differences between the stroke patients and the HCs, the sample size of 42 stroke patients analyzed in the current study was somewhat lacking in statistical power, and large sample size studies are needed for further demonstration. Second, it was reported that corticospinal tract lesion load was a significant predictor of motor deficit (Zhu et al., 2010; Feng et al., 2015). However, in the current study, we focused on investigating whether subcortical stroke patients would exhibit abnormal dynamic characteristics of brain activity relative to healthy controls and to investigate whether the altered dynamic regional indexes were associated with clinical behavior in stroke patients. Hence, here, we did not give much thought to the characteristics of diffusion-tensor imaging of the patients. Third, the correlation analyses cannot pass the FDR or Bonferroni correction. Larger sample size will be necessary to confirm the current results in the future studies. Fourth, physiological noise of cardiac and respiratory cycles was not monitored during the MRI scanning, which may influence brain activity alterations. It is possible that alterations in network dynamics may reflect changes in brain state, since few constraints were imposed on a participant's cognitive processes during the scanning. Finally, although the correlation analyses between dynamic regional indexes in regions showing significant group differences and size of lesion volume revealed no significant correlation, the heterogeneous clinical characteristics, such as lesion location, stroke severity and size of lesion volume, exhibited a relatively large variation across subjects and should be taken into consideration when interpreting the results.

\section{CONCLUSION}

The pattern of intrinsic brain activity variability in multiple brain networks is altered in stroke patients with motor deficits compared with healthy controls. The alterations of dynamic brain activity in the SMN and DMN were correlated with the degree of motor functional impairment. Resting-state fMRI dynamic regional indexes might be potential tools to assess stroke patients' motor function. Future studies will be needed to clarify the underlying mechanisms of alterations in the dynamic regional metrics after stroke.

\section{AUTHOR CONTRIBUTIONS}

JC contributed to the experiments, data analysis and writing of the manuscript. DS contributed to performing the experiments, and writing and revising the manuscript. YS contributed to the data collection. WJ designed the experiments and revised the manuscript. YW contributed to the data analysis and manuscript revision. QX contributed to the manuscript revision. CR is the guarantor of this study and had complete access to all data in the study. All authors are accountable for the contents of this research.

\section{FUNDING}

The study was supported by the National Natural Science Foundation of China (81571277).

\section{ACKNOWLEDGMENTS}

The authors thank all volunteers who participated in the study and the staff of the Med-X Research Institute and School of Biomedical Engineering Shanghai Jiao Tong University in Shanghai, China for their selfless and valuable assistance. 


\section{REFERENCES}

Abrams, D. A., Lynch, C. J., Cheng, K. M., Phillips, J., Supekar, K., Ryali, S., et al. (2013). Underconnectivity between voice-selective cortex and reward circuitry in children with autism. Proc. Natl. Acad. Sci. U.S.A. 110, 12060-12065. doi: 10.1073/pnas.1302982110

Allen, E. A., Damaraju, E., Plis, S. M., Erhardt, E. B., Eichele, T., and Calhoun, V. D. (2014). Tracking whole-brain connectivity dynamics in the resting state. Cereb. Cortex 24, 663-676. doi: 10.1093/cercor/bhs352

Andrews, S. C., Hoy, K. E., Enticott, P. G., Daskalakis, Z. J., and Fitzgerald, P. B. (2011). Improving working memory: the effect of combining cognitive activity and anodal transcranial direct current stimulation to the left dorsolateral prefrontal cortex. Brain Stimul. 4, 84-89. doi: 10.1016/j.brs.2010.06.004

Archer, D. B., Misra, G., Patten, C., and Coombes, S. A. (2016). Microstructural properties of premotor pathways predict visuomotor performance in chronic stroke. Hum. Brain Mapp. 37, 2039-2054. doi: 10.1002/hbm.23155

Ashburner, J. (2007). A fast diffeomorphic image registration algorithm. Neuroimage 38, 95-113. doi: 10.1016/j.neuroimage.2007.07.007

Badaut, J., Ashwal, S., Adami, A., Tone, B., Recker, R., Spagnoli, D., et al. (2011). Brain water mobility decreases after astrocytic aquaporin-4 inhibition using RNA interference. J. Cereb. Blood Flow Metab. 31, 819-831. doi: 10.1038/jcbfm. 2010.163

Biswal, B., Yetkin, F. Z., Haughton, V. M., and Hyde, J. S. (1995). Functional connectivity in the motor cortex of resting human brain using echo-planar MRI. Magn. Reson. Med. 34, 537-541. doi: 10.1002/mrm.1910340409

Calhoun, V. D., Kiehl, K. A., and Pearlson, G. D. (2008). Modulation of temporally coherent brain networks estimated using ICA at rest and during cognitive tasks. Hum. Brain Mapp. 29, 828-838. doi: 10.1002/hbm.20581

Carmichael, S. T., Wei, L., Rovainen, C. M., and Woolsey, T. A. (2001). New patterns of intracortical projections after focal cortical stroke. Neurobiol. Dis. 8, 910-922. doi: 10.1006/nbdi.2001.0425

Chang, E. F., Niziolek, C. A., Knight, R. T., Nagarajan, S. S., and Houde, J. F. (2013). Human cortical sensorimotor network underlying feedback control of vocal pitch. Proc. Natl. Acad. Sci. U.S.A. 110, 2653-2658. doi: 10.1073/pnas. 1216827110

Cunnington, R., Windischberger, C., Deecke, L., and Moser, E. (2003). The preparation and readiness for voluntary movement: a high-field event-related fMRI study of the Bereitschafts-BOLD response. Neuroimage 20, 404-412. doi: 10.1016/s1053-8119(03)00291-x

Damoiseaux, J. S., Rombouts, S. A., Barkhof, F., Scheltens, P., Stam, C. J., Smith, S. M., et al. (2006). Consistent resting-state networks across healthy subjects. Proc. Natl. Acad. Sci. U.S.A. 103, 13848-13853. doi: 10.1073/pnas.0601417103

Dancause, N., Barbay, S., Frost, S. B., Plautz, E. J., Chen, D., Zoubina, E. V., et al. (2005). Extensive cortical rewiring after brain injury. J. Neurosci. 25, 10167-10179. doi: 10.1523/JNEUROSCI.3256-05.2005

Duncan, E. S., and Small, S. L. (2017). Changes in dynamic resting state network connectivity following aphasia therapy. Brain Imaging Behav. 12, 1141-1149. doi: 10.1007/s11682-017-9771-2

Edwards, J. D., Kapoor, A., Linkewich, E., and Swartz, R. H. (2018). Return to work after young stroke: a systematic review. Int. J. Stroke 13, 243-256. doi: $10.1177 / 1747493017743059$

Feng, W., Wang, J., Chhatbar, P. Y., Doughty, C., Landsittel, D., Lioutas, V. A., et al. (2015). Corticospinal tract lesion load: an imaging biomarker for stroke motor outcomes. Ann. Neurol. 78, 860-870. doi: 10.1002/ana.24510

Ferrigno, M., Bricout, N., Leys, D., Estrade, L., Cordonnier, C., Personnic, T., et al. (2018). Intravenous recombinant tissue-type plasminogen activator: influence on outcome in anterior circulation ischemic stroke treated by mechanical thrombectomy. Stroke 49, 1377-1385. doi: 10.1161/STROKEAHA.118.020490

Fox, M. D., and Raichle, M. E. (2007). Spontaneous fluctuations in brain activity observed with functional magnetic resonance imaging. Nat. Rev. Neurosci. 8, 700-711. doi: 10.1038/nrn2201

Fu, Z., Tu, Y., Di, X., Du, Y., Pearlson, G. D., Turner, J. A., et al. (2017). Characterizing dynamic amplitude of low-frequency fluctuation and its relationship with dynamic functional connectivity: an application to schizophrenia. Neuroimage 180(Pt B), 619-631. doi: 10.1016/j.neuroimage. 2017.09.035

Gonzalez-Castillo, J., Hoy, C. W., Handwerker, D. A., Robinson, M. E., Buchanan, L. C., Saad, Z. S., et al. (2015). Tracking ongoing cognition in individuals using brief, whole-brain functional connectivity patterns. Proc. Natl. Acad. Sci. U.S.A. 112, 8762-8767. doi: 10.1073/pnas.1501242112

Grefkes, C., and Fink, G. R. (2014). Connectivity-based approaches in stroke and recovery of function. Lancet Neurol. 13, 206-216. doi: 10.1016/S1474-4422(13) 70264-3

Hillis, A. E., and Tippett, D. C. (2014). Stroke recovery: surprising influences and residual consequences. Adv. Med. 2014:378263. doi: 10.1155/2014/378263

Hindriks, R., Adhikari, M. H., Murayama, Y., Ganzetti, M., Mantini, D., Logothetis, N. K., et al. (2016). Corrigendum to "Can sliding-window correlations reveal dynamic functional connectivity in resting-state fMRI?”. Neuroimage 132:115. doi: 10.1016/j.neuroimage.2016.02.007

Li, Z., Kadivar, A., Pluta, J., Dunlop, J., and Wang, Z. (2012). Test-retest stability analysis of resting brain activity revealed by blood oxygen level-dependent functional MRI. J. Magn. Reson. Imaging 36, 344-354. doi: 10.1002/jmri. 23670

Liao, X., Yuan, L., Zhao, T., Dai, Z., Shu, N., Xia, M., et al. (2015). Spontaneous functional network dynamics and associated structural substrates in the human brain. Front. Hum. Neurosci. 9:478. doi: 10.3389/fnhum.7015.00478

Liu, F., Guo, W., Liu, L., Long, Z., Ma, C., Xue, Z., et al. (2013). Abnormal amplitude low-frequency oscillations in medication-naive, first-episode patients with major depressive disorder: a resting-state fMRI study. J. Affect. Disord. 146, 401-406. doi: 10.1016/j.jad.2012.10.001

Liu, F., Hu, M., Wang, S., Guo, W., Zhao, J., Li, J., et al. (2012). Abnormal regional spontaneous neural activity in first-episode, treatment-naive patients with latelife depression: a resting-state fMRI study. Prog. Neuropsychopharmacol. Biol. Psychiatry 39, 326-331. doi: 10.1016/j.pnpbp.2012.07.004

Liu, F., Wang, Y., Li, M., Wang, W., Li, R., Zhang, Z., et al. (2017). Dynamic functional network connectivity in idiopathic generalized epilepsy with generalized tonic-clonic seizure. Hum. Brain Mapp. 38, 957-973. doi: 10.1002/ hbm. 23430

Liu, G., Dang, C., Peng, K., Xie, C., Chen, H., Xing, S., et al. (2015). Increased spontaneous neuronal activity in structurally damaged cortex is correlated with early motor recovery in patients with subcortical infarction. Eur. J. Neurol. 22, 1540-1547. doi: 10.1111/ene. 12780

Liu, L., Wang, D., Wong, K. S., and Wang, Y. (2011). Stroke and stroke care in China: huge burden, significant workload, and a national priority. Stroke 42 , 3651-3654. doi: 10.1161/STROKEAHA.111.635755

Liu, T., Li, J., Huang, S., Li, C., Zhao, Z., Wen, G., et al. (2017). Altered restingstate functional activity in isolated pontine infarction patients with pathological laughing and crying. Oncotarget 8, 84529-84539. doi: 10.18632/oncotarget. 19307

Liu, Y., D’Arceuil, H., He, J., Duggan, M., Gonzalez, G., Pryor, J., et al. (2007). MRI of spontaneous fluctuations after acute cerebral ischemia in nonhuman primates. J. Magn. Reson. Imaging 26, 1112-1116. doi: 10.1002/jmri.21131

Logothetis, N. K., Pauls, J., Augath, M., Trinath, T., and Oeltermann, A. (2001). Neurophysiological investigation of the basis of the fMRI signal. Nature 412, 150-157. doi: $10.1038 / 35084005$

Lu, J., Liu, H., Zhang, M., Wang, D., Cao, Y., Ma, Q., et al. (2011). Focal pontine lesions provide evidence that intrinsic functional connectivity reflects polysynaptic anatomical pathways. J. Neurosci. 31, 15065-15071. doi: 10.1523/ JNEUROSCI.2364-11.2011

Mohamed, M. A., Yousem, D. M., Tekes, A., Browner, N., and Calhoun, V. D. (2004). Correlation between the amplitude of cortical activation and reaction time: a functional MRI study. AJR Am. J. Roentgenol. 183, 759-765. doi: 10. 2214/ajr.183.3.1830759

Murphy, K., Birn, R. M., Handwerker, D. A., Jones, T. B., and Bandettini, P. A. (2009). The impact of global signal regression on resting state correlations: are anti-correlated networks introduced? Neuroimage 44, 893-905. doi: 10.1016/j. neuroimage.2008.09.036

Nudo, R. J., and Masterton, R. B. (1990). Descending pathways of the spinal cord, III: sites of origin of the corticospinal tract. J. Comp. Neurol. 296, 559-583. doi: 10.1002/cne.902960405

Qiu, Y. W., Han, L. J., Lv, X. F., Jiang, G. H., Tian, J. Z., Zhuo, F. Z., et al. (2011). Regional homogeneity changes in heroin-dependent individuals: resting-state functional MR imaging study. Radiology 261, 551-559. doi: 10.1148/radiol. 11102466

Raichle, M. E. (2006). Neuroscience. The brain's dark energy. Science 314, 12491250. doi: $10.1126 /$ science. 1134405 
Sakoglu, U., Pearlson, G. D., Kiehl, K. A., Wang, Y. M., Michael, A. M., and Calhoun, V. D. (2010). A method for evaluating dynamic functional network connectivity and task-modulation: application to schizophrenia. MAGMA 23, 351-366. doi: 10.1007/s10334-010-0197-8

Shima, K., and Tanji, J. (1998). Both supplementary and presupplementary motor areas are crucial for the temporal organization of multiple movements. J. Neurophysiol. 80, 3247-3260. doi: 10.1152/jn.1998.80.6.3247

Skidmore, F. M., Yang, M., Baxter, L., von Deneen, K. M., Collingwood, J., He, G., et al. (2013). Reliability analysis of the resting state can sensitively and specifically identify the presence of Parkinson disease. Neuroimage 75, 249-261. doi: 10.1016/j.neuroimage.2011.06.056

Soddu, A., Vanhaudenhuyse, A., Bahri, M. A., Bruno, M. A., Boly, M., Demertzi, A., et al. (2012). Identifying the default-mode component in spatial IC analyses of patients with disorders of consciousness. Hum. Brain Mapp. 33, 778-796. doi: 10.1002/hbm.21249

Thompson, G. J., Magnuson, M. E., Merritt, M. D., Schwarb, H., Pan, W. J., McKinley, A., et al. (2013). Short-time windows of correlation between large-scale functional brain networks predict vigilance intraindividually and interindividually. Hum. Brain Mapp. 34, 3280-3298. doi: 10.1002/hbm.22140

Tsai, Y., Yuan, R., Huang, Y., Weng, H., Yeh, M., Lin, C., et al. (2014). Altered resting-state fMRI signals in acute stroke patients with ischemic penumbra. PLoS One 9:e105117. doi: 10.1371/journal.pone.0105117

Venketasubramanian, N., Lee, C. F., Young, S. H., Tay, S. S., Umapathi, T., Lao, A. Y., et al. (2017). Prognostic factors and pattern of long-term recovery with MLC601 (NeuroAiD) in the Chinese medicine neuroaid efficacy on stroke recovery - extension study. Cerebrovasc. Dis. 43, 36-42. doi: 10.1159/000452285

Wang, L., Yu, C., Chen, H., Qin, W., He, Y., Fan, F., et al. (2010). Dynamic functional reorganization of the motor execution network after stroke. Brain 133, 1224-1238. doi: 10.1093/brain/awq043

Ween, J. E. (2008). Functional imaging of stroke recovery: an ecological review from a neural network perspective with an emphasis on motor systems. J. Neuroimaging 18, 227-236. doi: 10.1111/j.1552-6569.2007.00180.x

Wu, J., Quinlan, E. B., Dodakian, L., McKenzie, A., Kathuria, N., Zhou, R. J., et al. (2015). Connectivity measures are robust biomarkers of cortical function and plasticity after stroke. Brain 138, 2359-2369. doi: 10.1093/brain/awv156

Yan, C. G., Yang, Z., Colcombe, S. J., Zuo, X. N., and Milham, M. P. (2017). Concordance among indices of intrinsic brain function: insights from interindividual variation and temporal dynamics. Sci. Bull. 23, 1572-1584. doi: 10. 1016/j.scib.2017.09.015

Yang, G., Wang, Y., Zeng, Y., Gao, G. F., Liang, X., Zhou, M., et al. (2013). Rapid health transition in China, 1990-2010: findings from the global burden of disease study 2010. Lancet 381, 1987-2015. doi: 10.1016/S0140-6736(13) 61097-1
Yin, D., Luo, Y., Song, F., Xu, D., Peterson, B. S., Sun, L., et al. (2013). Functional reorganization associated with outcome in hand function after stroke revealed by regional homogeneity. Neuroradiology 55, 761-770. doi: 10.1007/s00234013-1146-9

Yip, E., Yun, J., Wachowicz, K., Gabos, Z., Rathee, S., and Fallone, B. G. (2017). Sliding window prior data assisted compressed sensing for MRI tracking of lung tumors. Med. Phys. 44, 84-98. doi: 10.1002/mp.12027

Yuan, C., Zhu, H., Ren, Z., Yuan, M., Gao, M., Zhang, Y., et al. (2018). Precuneusrelated regional and network functional deficits in social anxiety disorder: a resting-state functional MRI study. Compr. Psychiatry 82, 22-29. doi: 10.1016/j. comppsych.2017.12.002

Zang, Y., Jiang, T., Lu, Y., He, Y., and Tian, L. (2004). Regional homogeneity approach to fMRI data analysis. Neuroimage 22, 394-400. doi: 10.1016/j. neuroimage.2003.12.030

Zang, Y. F., He, Y., Zhu, C. Z., Cao, Q. J., Sui, M. Q., Liang, M., et al. (2007). Altered baseline brain activity in children with ADHD revealed by resting-state functional MRI. Brain Dev. 29, 83-91. doi: 10.1016/j.braindev.2006.07.002

Zhang, S., Ide, J. S., and Li, C. S. (2012). Resting-state functional connectivity of the medial superior frontal cortex. Cereb. Cortex 22, 99-111. doi: 10.1093/cercor/ bhr088

Zhang, Y., Wang, L., Yang, J., Yan, R., Zhang, J., Sang, L., et al. (2017). Abnormal functional networks in resting-state of the sub-cortical chronic stroke patients with hemiplegia. Brain Res. 1663, 51-58. doi: 10.1016/j.brainres.2017. 02.012

Zhu, J., Jin, Y., Wang, K., Zhou, Y., Feng, Y., Yu, M., et al. (2015). Frequencydependent changes in the regional amplitude and synchronization of restingstate functional MRI in stroke. PLoS One 10:e0123850. doi: 10.1371/journal. pone. 0123850

Zhu, L. L., Lindenberg, R., Alexander, M. P., and Schlaug, G. (2010). Lesion load of the corticospinal tract predicts motor impairment in chronic stroke. Stroke 41, 910-915. doi: 10.1161/STROKEAHA.109.577023

Conflict of Interest Statement: The authors declare that the research was conducted in the absence of any commercial or financial relationships that could be construed as a potential conflict of interest.

Copyright (c) 2019 Chen, Sun, Shi, Jin, Wang, Xi and Ren. This is an open-access article distributed under the terms of the Creative Commons Attribution License (CC BY). The use, distribution or reproduction in other forums is permitted, provided the original author(s) and the copyright owner(s) are credited and that the original publication in this journal is cited, in accordance with accepted academic practice. No use, distribution or reproduction is permitted which does not comply with these terms. 